\title{
GENERATION AND PROPAGATION OF NONLINEAR WAVES IN A TOWING TANK
}

\author{
Marcin Drzewiecki ${ }^{1,2}$ \\ Wojciech Sulisz ${ }^{3}$ \\ ${ }^{1}$ Gdańsk University of Technology, Poland \\ ${ }^{2}$ Ship Deign and Research Centre CTO S.A., Gdańsk, Poland \\ ${ }^{3}$ Institute of Hydroengineering of Polish Academy of Sciences IBW PAN, Gdańsk, Poland
}

\begin{abstract}
The paper presents the results of the research focused on linear and nonlinear wave generation and propagation in a deepwater towing tank equipped with a single flap-type wavemaker of variable draft. The problem of wave generation and propagation has been theoretically formulated and solved by applying an analytical method; linear and nonlinear solutions were obtained. The linear solution has been verified experimentally. The laboratory experiments confirmed that a linear model can be applied to predict the generation and propagation of water waves of low steepness. However, according to the analysis, the discrepancies between wave profiles predicted by applying the linear and nonlinear models rapidly increases with increasing wave steepness. Additionally, the secondary phenomena which occur in the towing tank, including: disintegration of wave profile, wave reflections from the beach and wave damping, were analyzed. Knowledge on the nonlinear processes and phenomena is essential for modeling the environmental conditions during tests carried out to secure the safety of the naval and offshore constructions. The theoretical formulation was derived and the solution was obtained by the Institute of Hydroengineering of the Polish Academy of Sciences IBW PAN while the experimental research was carried out in Ship Hydromechanics Division of the Ship Design and Research Centre CTO S.A.
\end{abstract}

Keywords: wavemaker, towing tank, nonlinear waves, waves generation, waves propagation

\section{INTRODUCTION}

Physical modeling of the ocean hydrodynamics in towing tanks is vital for a sustainable development of naval architecture and offshore sectors. The understanding of the physics of the generation and propagation of nonlinear water waves in a towing tank is of fundamental importance for both scientists and engineers.

Numerous theoretical studies were conducted to describe the generation and propagation of waves in a hydrodynamic laboratory. Linear wavemaker theories were derived by Havelock [7] and Biesel and Suquet [1]. Laboratory experiments carried out in wave flumes confirmed suitability of the linear wave theory to mechanically generated waves of low steepness [19, 5, 9].

Research carried out in the Laboratório Nacional de Engenharia Civil in Lisbon, has shown that linear wavemaker theory is correct for regular waves investigated there [2]. However, for irregular waves, the measured transfer function was inconsistent with linear wavemaker theory. The authors indicate that it may arise from nonlinearities and energy losses.

The development of weakly-nonlinear wavemaker theories allowed for extending the range of applicability to steeper waves and gave insight into the physics of processes and side effects of the wave generation in a wave flume $[12,8,16,13,15]$. More complex numerical techniques were used to simulate nonlinear wave generation $[14,6,10,21,22,11]$. The problem is that up to now, theoretical investigations have focused on the modelling of waves in typical wave flumes. Far less attention has been devoted to the problem of the generation and propagation of waves in towing tanks that in general are much longer and wider than flumes. The specific features of generation systems applied in towing tanks require special 
attention and more advanced studies. The derived solutions have numerous limitations and drawbacks, and may not be applicable to explain many phenomena observed in towing tanks; this fact motivated the present study.

In this work, a theoretical approach is applied to predict the generation and propagation of nonlinear water waves in a towing tank. First, a boundary-value problem is formulated to describe the generation of nonlinear water waves in a towing tank and is solved by applying an analytical method. Then, the solution is applied to analyze the effect of wave frequencies and wave steepness on the generation and propagation of water waves in a long towing tank. Special attention is devoted to the magnitude of nonlinear wave components. Finally, theoretical results are compared with experimental data.

\section{THEORETICAL FORMULATION}

\section{GOVERNING EQUATIONS}

The problem of the generation and propagation of nonlinear water waves in a towing tank is considered. A right-hand Cartesian coordinate system is selected such that the $x y$ plane is horizontal and coincides with the undisturbed free surface and $z$ points vertically upwards, Fig. 1 . It is assumed that the fluid is inviscid and incompressible, and that the fluid motion is irrotational. Moreover, it is assumed that the horizontal bottom and the vertical-side walls are impervious.

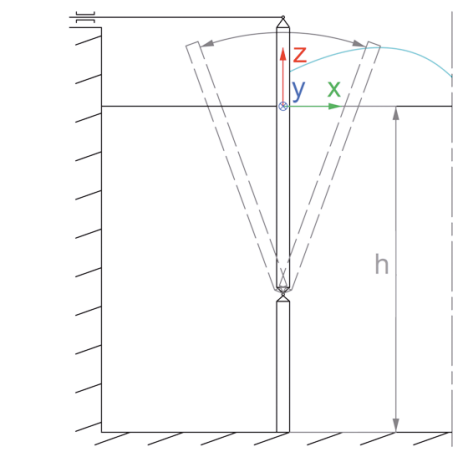

Fig. 1. Cartesian coordinate system in a towing tank for the considered problem

According to the assumptions, the velocity vector, $V(x, z, t)$, may be computed from a velocity potential $\Phi(x, z, t)$

$$
V=\nabla \Phi(x, z, t)
$$

where $\nabla(\cdot)$ is the two-dimensional vector differential operator. The fluid motion is governed by the continuity equation

and the Bernoulli equation

$$
\nabla^{2} \Phi=0
$$

$$
\Phi_{t}+\frac{1}{\rho} P+g z+\frac{1}{2}|\nabla \Phi|^{2}=B
$$

where $\rho$ is the fluid mass density, $P$ is the dynamic pressure, $g$ is the acceleration due to gravity, and $B$ is the Bernoulli constant.

The velocity potential, $V(x, z, t)$, satisfies the Laplace equation

$$
\nabla^{2} \Phi=0
$$

At the free surface, the velocity potential, $V(x, z, t)$, has to satisfy the combined free-surface boundary condition

$$
\begin{aligned}
& \Phi_{t t}+g \Phi_{z}+\left(|\nabla \Phi|^{2}\right)_{t} \\
& +\frac{1}{2} \nabla \Phi \cdot \nabla|\nabla \Phi|^{2}-B_{t}=0, \\
& \mathrm{z}=\eta(x, t)
\end{aligned}
$$

and the dynamic boundary condition

$$
\Phi_{t}+g \eta+\frac{1}{2}|\nabla \Phi|^{2}-B=0, z=\eta(x, t)
$$

At the wavemaker, the kinematic wavemaker boundary condition must be satisfied:

$$
\chi_{t}+\Phi_{z} \chi_{z}-\Phi_{x}=0, \quad x=\chi(z, t)
$$

At the sea bottom, the following boundary condition must be satisfied:

$$
\Phi_{z}=0, \quad z=-h
$$

Moreover, the velocity potential must satisfy boundary conditions at infinity and initial conditions [20]. The boundary conditions at infinity state that the first-order velocity potential at $x \rightarrow \pm \infty$ represents only outgoing waves.

\section{PERTURBATION METHOD}

The difficulties associated with an unknown location where the free-surface boundary conditions must be satisfied may be overcome by expanding the free-surface boundary conditions in a Taylor series about a known location that is assumed to be the undisturbed free surface $z=0$. A similar procedure is applied to the kinematic wavemaker boundary condition. Accordingly, the combined free-surface boundary condition, Eq. (3b), the dynamic free-surface boundary condition, Eq. (3c), and the kinematic wavemaker boundary condition, Eq. (3d), may be written as follows

$$
\begin{aligned}
& \sum_{n=0}^{\infty} \frac{\eta^{n}}{n !} \frac{\partial^{n}}{\partial z^{n}} \\
& \cdot\left(\Phi_{t t}+g \Phi_{z}+\left(|\nabla \Phi|^{2}\right)_{t}+\frac{1}{2} \nabla \Phi \cdot \nabla|\nabla \Phi|^{2}-B_{t}\right), \\
& =0, \quad \mathrm{z}=0
\end{aligned}
$$

$$
\sum_{n=0}^{\infty} \frac{\eta^{n}}{n !} \frac{\partial^{n}}{\partial z^{n}}\left(\Phi_{t}+g \eta+\frac{1}{2}|\nabla \Phi|^{2}-B\right)=0
$$




$$
\begin{aligned}
& \sum_{n=0}^{\infty} \frac{\chi^{n}}{n !} \frac{\partial^{n}}{\partial x^{n}}\left(\chi_{t}+\Phi_{z} \chi_{z}-\Phi_{x}\right)=0, \\
& x=0
\end{aligned}
$$

which usually helps to obtain a solution [8].

By applying Eq. (4), the boundary-value problem for the velocity potential, Eq. (3), may be written in the following form

$$
\nabla^{2} \Phi=0
$$

with boundary conditions

$$
\begin{gathered}
\Phi_{t t}+g \Phi_{z}+\left(|\nabla \Phi|^{2}\right)_{t}+ \\
\frac{1}{2} \nabla \Phi \cdot \nabla|\nabla \Phi|^{2}-B_{t} \\
+\eta\left(\Phi_{t t}+g \Phi_{z}+\left(|\nabla \Phi|^{2}\right)_{t}+\frac{1}{2} \nabla \Phi \cdot \nabla|\nabla \Phi|^{2}-B_{t}\right)_{z} \\
+\ldots=0, \quad \mathrm{z}=0 \\
\Phi_{t}+g \eta+\frac{1}{2}|\nabla \Phi|^{2}-B \\
+\eta\left(\Phi_{t}+g \eta+\frac{1}{2}|\nabla \Phi|^{2}-B\right)_{z} \\
+\ldots=0, \quad z=0 \\
\chi_{t}+\Phi_{z} \chi_{z}-\Phi_{x}+\chi\left(\chi_{t}+\Phi_{z} \chi_{z}-\Phi_{x}\right)_{x} \\
+\ldots=0, \quad x=0 \\
\Phi_{z}=0, \quad z=-h
\end{gathered}
$$

Moreover, the velocity potential $\Phi$, must satisfy boundary conditions at infinity.

An analytical solution to Eq. (5) may be found in the frame of weakly nonlinear wave theory. In order to obtain a solution, it is assumed that the quantities $\Phi, \eta, P$, and $B$ may be written in the form of the following series

$$
\begin{gathered}
\Phi(x, z, t)={ }_{1} \Phi+{ }_{2} \Phi+\ldots, \\
\eta(x, t)={ }_{0} \eta+{ }_{1} \eta+{ }_{2} \eta+\ldots, \\
P(x, z, t)={ }_{0} P+{ }_{1} P+{ }_{2} P+\ldots,
\end{gathered}
$$

and

$$
B(t)={ }_{1} B+{ }_{2} B+\ldots,
$$

where a quantity with a left subscript $n, n=0,1, \ldots$ is of the order of $\left(A_{1} k_{1}\right)^{\mathrm{n}}, A_{1} k_{1} \ll 1, k_{1}$ in which $A_{1}$ is the characteristic wave amplitude, $k_{1}$ is the characteristic wave number.

By substituting the formulas to the original boundaryvalue problem and collecting terms of the same order in wave steepness one obtains a set of the boundary-value problems with boundary conditions at $z=0$ and $x=0$.

$$
\begin{array}{r}
\nabla_{1}^{2} \Phi=0, \quad-h \leq z \leq 0 \\
{ }_{1} \Phi_{t t}+g_{1} \Phi_{z}-{ }_{1} B_{t}=0, \quad z=0 \\
\Phi_{t t}+g_{1} \Phi_{z}-{ }_{1} B_{t}=0, \quad z=0
\end{array}
$$

Moreover, the velocity potential must satisfy boundary conditions at infinity.

The boundary-value problem for the second-order velocity potential can be written in the following form

$$
\begin{gathered}
\nabla_{2}^{2} \Phi=0, \quad-h \leq z \leq 0 \\
{ }_{2} \Phi_{t t}+g_{2} \Phi_{z}-{ }_{2} B_{t}=-\left(\left|\nabla_{1} \Phi\right|^{2}\right)_{t} \\
\left.-{ }_{1} \eta_{1} \Phi_{t t}+g_{1} \Phi_{z}\right), \quad z=0 \\
{ }_{2} \Phi_{x}={ }_{1} \Phi_{z} \chi_{z}-\chi_{1} \Phi_{x x}, \quad x=0 \\
{ }_{2} \Phi_{z}=0, \quad z=-h
\end{gathered}
$$

Moreover, the velocity potential must satisfy boundary conditions at infinity.

\section{SOLUTION}

\section{LINEAR SOLUTION}

The boundary-value problems, Eq. (7), for an excitation provided by simple-harmonic waves, may be solved in the present form. However, due to phase shift, it is more convenient to introduce spatial functions and separate a time-dependent factor. In order to conduct the separation, complex-valued spatial functions are introduced according to

$$
\begin{aligned}
{ }_{1} \Phi(x, z, t) & =\operatorname{Re}\left[{ }_{1} \phi(x, z) e^{-i \omega t}\right], \\
{ }_{1} \eta(x, t) & =\operatorname{Re}\left[\zeta(x) e^{-i \omega t}\right], \\
{ }_{1} P(x, z, t) & =\operatorname{Re}\left[{ }_{1} p(x, z) e^{-i \omega t}\right], \\
{ }_{1} B & =\operatorname{Re}(b),
\end{aligned}
$$

where Re denotes real part and $i=\sqrt{ }-1$.

The spatial wave-field components may be written in the following form:

$$
\begin{gathered}
{ }_{1} \phi(x, z)=-\frac{i g}{\omega} \sum_{m=1} A_{1 m} \frac{\cos \alpha_{1 m}(z+h)}{\cos \alpha_{1 m} h} e^{-\alpha{ }_{1 m} x}, \\
{ }_{1} \zeta(x)=\sum_{m=1}^{\infty} A_{1 m} e^{-\alpha_{1 m} x},
\end{gathered}
$$

where $A_{11}$ is the amplitude of the progressive wave and $A_{1 m}$, $\mathrm{m}=2,3, \ldots$, are the amplitudes of evanescent modes. The eigenvalues must satisfy the following relations:

$$
\frac{\omega^{2}}{g}=-\alpha_{1 m} \tan \alpha_{1 m} h, m \geq 1,
$$

where the eigenvalues are $\alpha_{1 m}=\left\{-i k_{1}, \alpha_{12}, \alpha_{13}, \ldots ; k_{1}, \alpha_{12}, \ldots>0\right\}$. 
The coefficients of the eigenfunction expansions can be quantified from the kinematic wavemaker boundary condition

$$
\begin{gathered}
A_{1 m}=\frac{4 \alpha_{1 m} \sin \alpha_{1 m} h}{\alpha_{1 m} h+\sin 2 \alpha_{1 m} h} \\
\cdot \int_{-h}^{0} \hat{\chi} \cos \alpha_{1 m}(z+h) d z
\end{gathered}
$$

The linear transfer function, according to (10d), has been calculated for the considered wavemaker in the deepwater towing tank. It has been calculated for three possible water levels - $5.19 \mathrm{~m}, 5.69 \mathrm{~m}, 6.19 \mathrm{~m}$ - and shown in, respectively, Fig. 2, Fig. 3, Fig. 4

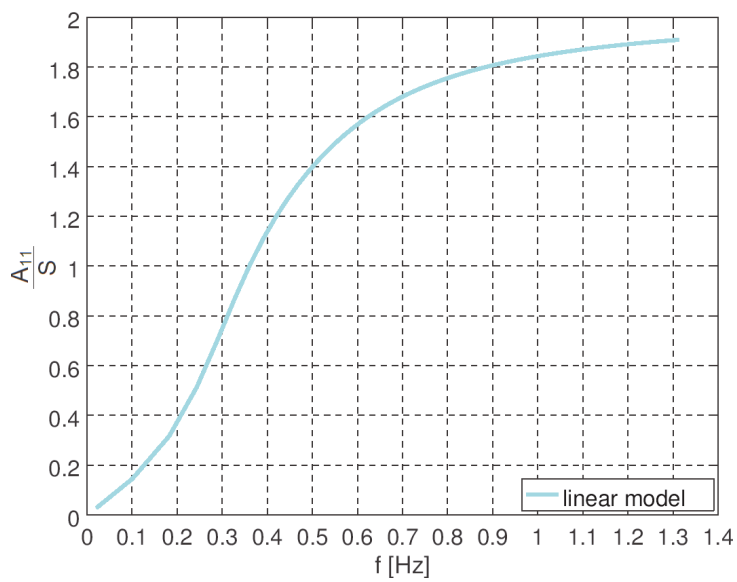

Fig. 2. Linear transfer function for a hinged wavemaker, $h=5.19 \mathrm{~m}$

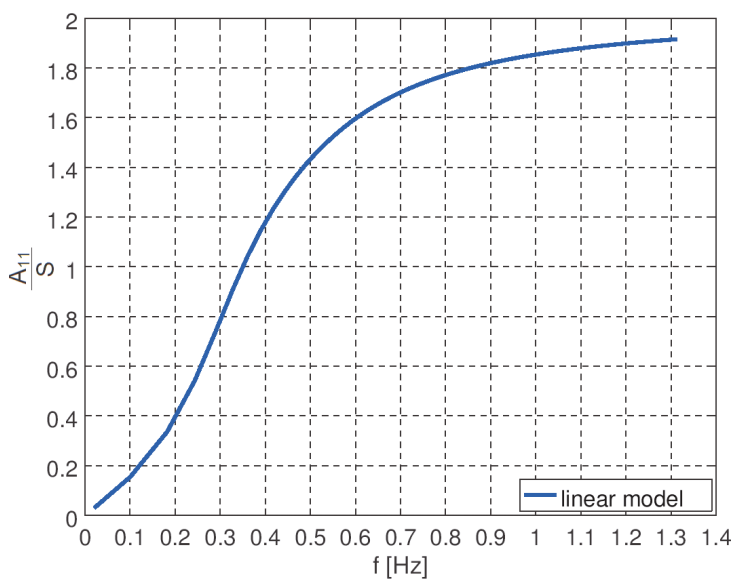

Fig. 3. Linear transfer function for a hinged wavemaker, $h=5.69 \mathrm{~m}$

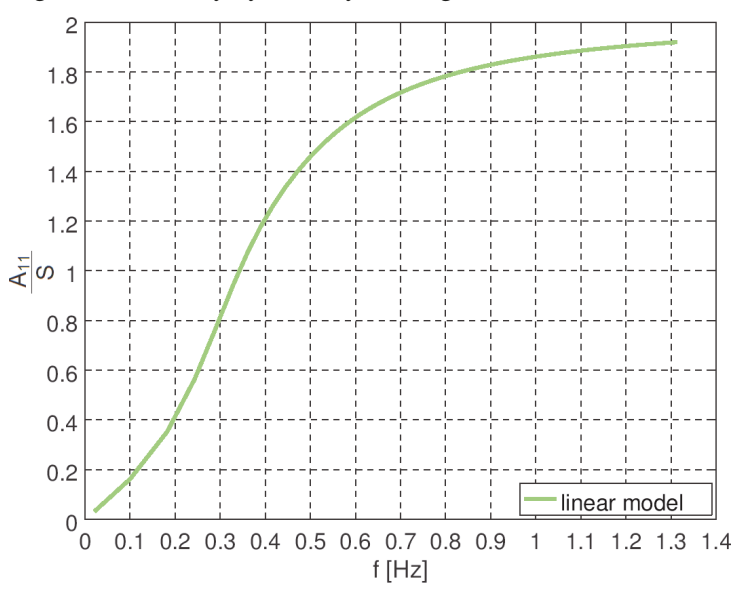

Fig. 4. Linear transfer function for a hinged wavemaker, $h=6.19 \mathrm{~m}$

\section{NONLINEAR SOLUTION}

It is convenient to solve the boundary-value problems, Eq. (8), by introducing spatial functions and separate a timedependent factor [17]. In order to conduct the separation of time complex-valued spatial functions are introduced according to

$$
\begin{aligned}
& { }_{2} \Phi(x, z, t)=\operatorname{Re}\left[{ }_{2} \phi(x, z) e^{-2 i \omega t}\right]+{ }_{2}^{0} \Phi(x, z), \\
& { }_{2} \eta(x, t)=\operatorname{Re}\left[{ }_{2} \varsigma(x) e^{-2 i \omega t}\right]+{ }_{2}^{0} \eta(x), \\
& { }_{2} \eta(x, t)=\operatorname{Re}\left[{ }_{2} \varsigma(x) e^{-2 i \omega t}\right]+{ }_{2}^{0} \eta(x),
\end{aligned}
$$

and

$$
{ }_{2} B=\operatorname{Re}\left({ }_{2} b\right) \text {. }
$$

The nonlinear components of the velocity potential and freesurface elevation may be expressed as follows

$$
\begin{aligned}
& { }_{2} \phi=-\frac{i g}{2 \omega} \sum_{j=1}^{\infty} A_{2 j} \frac{\cos \alpha_{2 j}(z+h)}{\cos \alpha_{2 j} h} e^{-\alpha_{2 j} x} \\
& -i \omega \sum_{m=1} \sum_{s=1} A_{1 m} A_{1 s} \frac{\alpha_{1 m} \alpha_{s m}}{4 \omega^{4} / g^{2}} \\
& \cdot \frac{6 \omega^{4} / g^{2}+4 \alpha_{1 m} \alpha_{1 s}+\alpha_{1 m}^{2}+\alpha_{1 s}^{2}}{4 \omega^{4} / g^{2}+\left(\alpha_{1 m}-\alpha_{1 s}\right)^{2}} \\
& \cdot \frac{\cos \left(\alpha_{1 m}+\alpha_{s m}\right)(z+h)}{\cos \alpha_{1 m} h \cos \alpha_{1 s} h} e^{-\left(\alpha_{1 m}+\alpha_{1 s}\right) x},
\end{aligned}
$$

$$
\begin{aligned}
& { }_{2} \zeta(x)=\sum_{j=1}^{\infty} A_{2 j} e^{-\alpha_{2 j} x}+\frac{g}{4 \omega^{2}} \sum_{m=1}^{\infty} \sum_{s=1}^{\infty} A_{1 m} A_{1 s} \\
& \cdot \frac{\left(\alpha_{1 m}+\alpha_{1 s}\right)^{2}\left(\omega^{4} / g^{2}+3 \alpha_{1 m} \alpha_{1 s}\right)}{4 \omega^{4} / g^{2}+\left(\alpha_{1 m}-\alpha_{1 s}\right)^{2}} e^{-\left(\alpha_{1 m}+\alpha_{1 s}\right) x},
\end{aligned}
$$

where $A_{21}$ is the amplitude of the free nonlinear wave, $A_{2 j}$, $j=2,3, \ldots$, are the amplitudes of the nonlinear components of evanescent modes.

The eigenvalues must satisfy the following relations

$$
\frac{4 \omega^{2}}{g}=-\alpha_{2 j} \tan \alpha_{2 j} h, j \geq 1
$$

where $\alpha_{2 j}=\left\{-i k_{2}, \alpha_{22}, \alpha_{23}, \ldots ; k_{2}, \alpha_{22}, \ldots>0\right\}$.

The nonlinear velocity potential consists of two main parts. The first part is associated with the summation over $\mathrm{j}$ and satisfies the homogeneous form of the combined free-surface boundary condition. The second part represents ${ }_{2} \phi^{F}$ and satisfies the nonhomogeneous form of the combined free-surface boundary condition $[17,18]$.

The coefficients of the eigenfunction expansions can be quantified from the wavemaker kinematic boundary condition

$A_{2 j}=\frac{4 \alpha_{2 j} \sin \alpha_{2 j} h}{\alpha_{2 j} h+\sin 2 \alpha_{2 j} h} \int_{-h}^{0}\left[-{ }_{2} \phi_{z}^{F}+\left({ }_{1} \phi_{z} \hat{\chi}\right)_{z}\right] \cos \alpha_{2 j}(z+h) d z$, $x=0$ 
In accordance with the linear and nonlinear solutions, simulations were carried out for the considered type of wavemaker.

The simulations included calculations of amplitudes: progressive wave $A_{11}$, undesirable free nonlinear wave $A_{21}$, resulting from using single flap-type wavemaker, and Stokes wave $A_{2 S}$ that accompanies the first harmonic. The calculations

Tab. 1. Linear and nonlinear wave components in the towing tank, $h=5.19 \mathrm{~m}$

\begin{tabular}{|c|c|c|}
\hline$k h$ & $\frac{\left|A_{21}\right|}{A_{11}}$ & $\frac{\left|A_{21}\right|}{A_{2 S}}$ \\
\hline 0.1 & 0.71 & 0.96 \\
\hline 0.2 & 0.31 & 0.83 \\
\hline 0.3 & 0.16 & 0.61 \\
\hline 0.4 & 0.06 & 0.30 \\
\hline 0.5 & 0.05 & 0.28 \\
\hline 0.6 & 0.13 & 0.83 \\
\hline 0.7 & 0.23 & 1.49 \\
\hline 0.8 & 0.32 & 2.17 \\
\hline 0.9 & 0.41 & 2.75 \\
\hline 1.0 & 0.48 & 3.17 \\
\hline 1.1 & 0.54 & 3.41 \\
\hline 1.2 & 0.58 & 3.50 \\
\hline 1.3 & 0.60 & 3.47 \\
\hline 1.4 & 0.62 & 3.36 \\
\hline 1.5 & 0.63 & 3.19 \\
\hline 1.6 & 0.64 & 2.99 \\
\hline 1.7 & 0.64 & 2.78 \\
\hline 1.8 & 0.63 & 2.56 \\
\hline
\end{tabular}

Tab. 2. Linear and nonlinear wave components in the towing tank, $h=5.69 \mathrm{~m}$

\begin{tabular}{|c|c|c|}
\hline$k h$ & $\frac{\left|A_{21}\right|}{A_{11}}$ & $\frac{\mid A_{21}}{A_{2 S}}$ \\
\hline 0.1 & 0.76 & 0.96 \\
\hline 0.2 & 0.34 & 0.84 \\
\hline 0.3 & 0.18 & 0.63 \\
\hline 0.4 & 0.08 & 0.35 \\
\hline 0.5 & 0.04 & 0.21 \\
\hline 0.6 & 0.12 & 0.68 \\
\hline 0.7 & 0.21 & 1.28 \\
\hline 0.8 & 0.30 & 1.89 \\
\hline 0.9 & 0.38 & 2.42 \\
\hline 1.0 & 0.45 & 2.79 \\
\hline 1.1 & 0.50 & 3.00 \\
\hline 1.2 & 0.54 & 3.08 \\
\hline 1.3 & 0.56 & 3.04 \\
\hline 1.4 & 0.58 & 2.94 \\
\hline 1.5 & 0.58 & 2.79 \\
\hline 1.6 & 0.58 & 2.61 \\
\hline 1.7 & 0.58 & 2.41 \\
\hline 1.8 & 0.58 & 2.22 \\
\hline
\end{tabular}

have been carried out for $k h$ values from 0.1 to 1.8 with increments of 0.1 and for three possible water levels $-5.19 \mathrm{~m}$, $5.69 \mathrm{~m}, 6.19 \mathrm{~m}$. The $k h$ is an multiplication of the wave number $k$ and water level $h$. The comparison between the amplitudes of nonlinear waves: $A_{21}, A_{2 \mathrm{~S}}$ with amplitudes of linear waves $A_{11}$ are presented in Tab. 1, Tab. 2, Tab. 3 and in Fig. 5, Fig. 6, Fig. 7, respectively.

Tab. 3. Linear and nonlinear wave components in the towing tank, $h=6.19 \mathrm{~m}$

\begin{tabular}{|c|c|c|}
\hline$k h$ & $\frac{\left|A_{21}\right|}{A_{11}}$ & $\frac{\left|A_{21}\right|}{A_{2 S}}$ \\
\hline 0.1 & 0.80 & 0.96 \\
\hline 0.2 & 0.36 & 0.85 \\
\hline 0.3 & 0.19 & 0.66 \\
\hline 0.4 & 0.09 & 0.38 \\
\hline 0.5 & 0.04 & 0.18 \\
\hline 0.6 & 0.10 & 0.57 \\
\hline 0.7 & 0.19 & 1.13 \\
\hline 0.8 & 0.28 & 1.69 \\
\hline 0.9 & 0.36 & 2.17 \\
\hline 1.0 & 0.42 & 2.51 \\
\hline 1.1 & 0.47 & 2.70 \\
\hline 1.2 & 0.50 & 2.77 \\
\hline 1.3 & 0.53 & 2.74 \\
\hline 1.4 & 0.54 & 2.64 \\
\hline 1.5 & 0.54 & 2.49 \\
\hline 1.6 & 0.54 & 2.33 \\
\hline 1.7 & 0.54 & 2.16 \\
\hline 1.8 & 0.53 & 1.98 \\
\hline & & \\
\hline & & \\
\hline
\end{tabular}

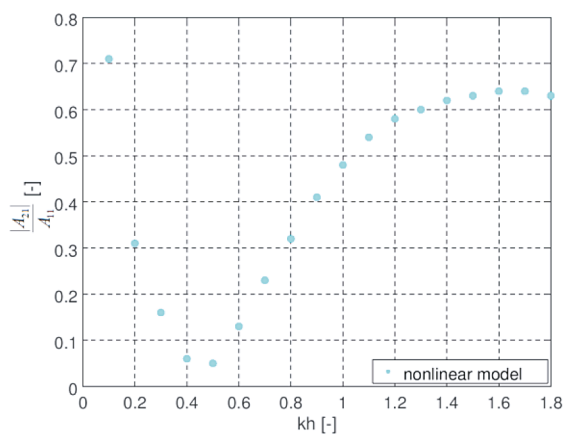

Fig. 5. Linear and nonlinear wave components in the towing tank, $h=5.19 \mathrm{~m}$

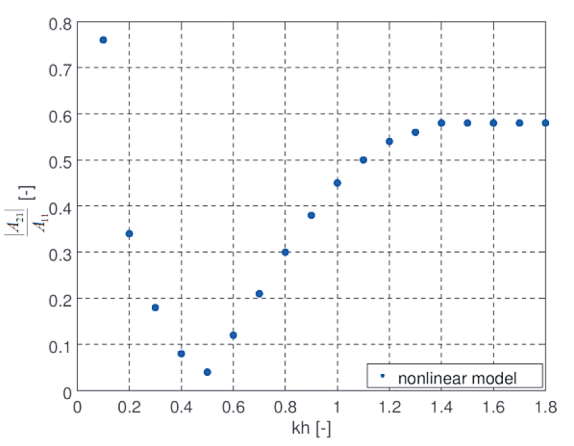

Fig. 6. Linear and nonlinear wave components in the towing tank, $h=5.69 \mathrm{~m}$ 


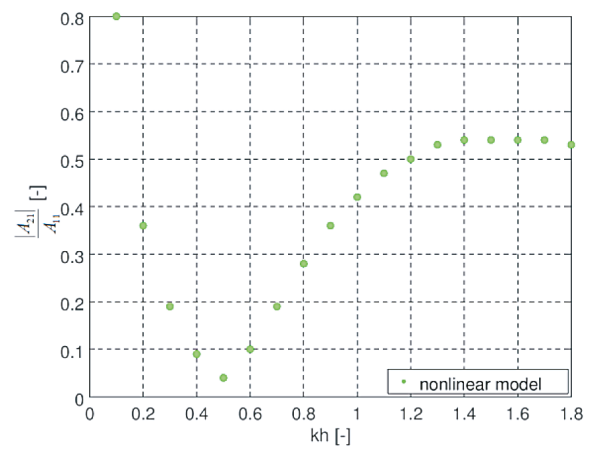

Fig. 7. Linear and nonlinear wave components in the towing tank, $h=6.19 \mathrm{~m}$

\section{VALIDATION}

\section{LABORATORY FACILITIES}

The proposed linear solution (10d) has been validated for deepwater towing tank of the following dimensions: length $270 \mathrm{~m}$, width $12 \mathrm{~m}$ and depth $6 \mathrm{~m}$, equipped with a single flap-type wavemaker, shown in Fig. 8.

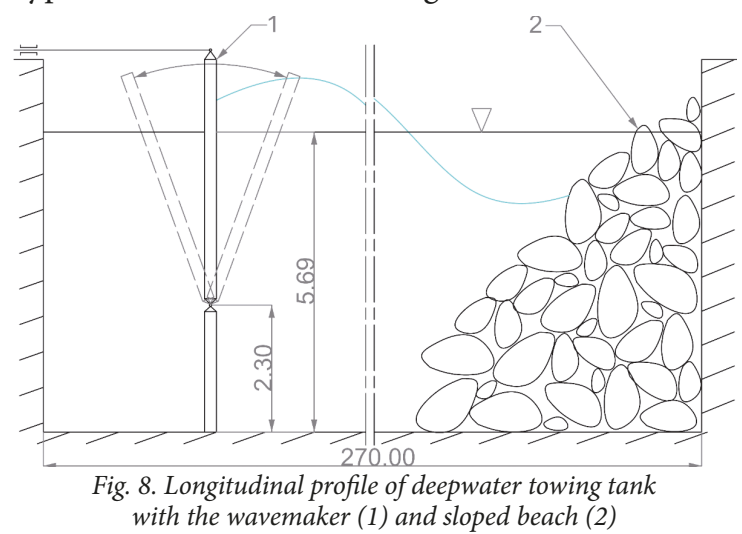

The wavemaker and deepwater towing tank of the Ship Design and Research Centre CTO S.A. are used for testing models of ships (Fig. 9) and anchored or bottom-fixed offshore structures e.g. oil rigs or wind turbines.

The model tests are carried out to predict the response of a structure to extreme sea state conditions.

The wavemaker is a position-controlled single flap-type device articulated above the channel bottom. It is equipped with hydraulically driven mechanism.

The wavemaker control system was modernized in 2015 - an analog control system was replaced by a new digital control system [3] further optimized in 2017 [4].

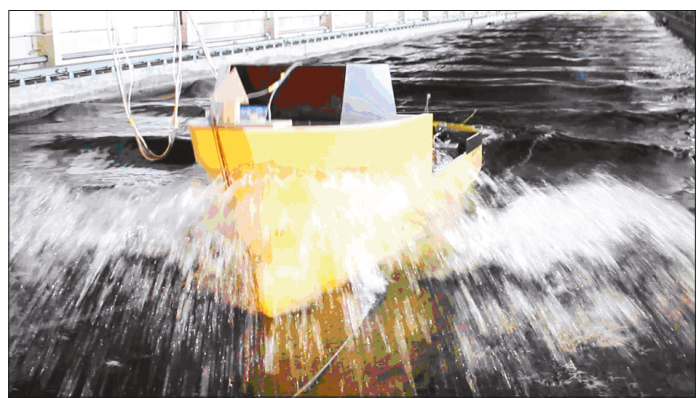

Fig. 9. The example seakeeping tests in CTO S.A.

\section{THE EXPERIMENTS}

The validation was based on the measurements of the flap displacements and wave profiles. The validation of the linear solution was carried out for regular waves of $5 \mathrm{~cm}$ height and frequencies from $0.3 \mathrm{~Hz}$ to $1.2 \mathrm{~Hz}$ with increments of $0.1 \mathrm{~Hz}$. The water depth in the towing tank was equal to $5.69 \mathrm{~m}$. The waves were measured using a wave gauge manufactured at CTO S.A. (Fig. 10). The wave gauge was mounted in the towing tank at the distance of $60 \mathrm{~m}$ from the flap. The displacements of the flap during wave generation were measured using linear displacement sensor WDS-1000-P60-SR-U manufactured by MicroEpsilon.

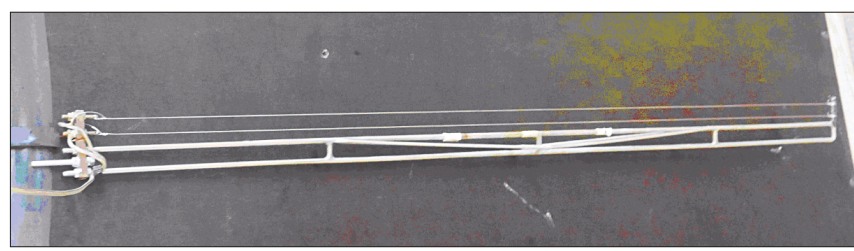

Fig. 10. Resistive wave gauge manufactured at CTO S.A.

Experimental results were compared with theoretical results. Due to idealization of the theoretical model, particular features of the actual towing tank are not taken into account in the presented formulation, e.g. flow damping between the wavemaker flap and towing tank walls as well as damping in waveguides area. This type of damping is out of scope of the present study. For that reason, a constant correction parameter $C p$ is introduced, evaluated so as to obtain the best match between the theoretical and experimental results. The resulting value is $C p=0.8$.

The comparison between theoretical results and experimental data is presented in Fig. 11.

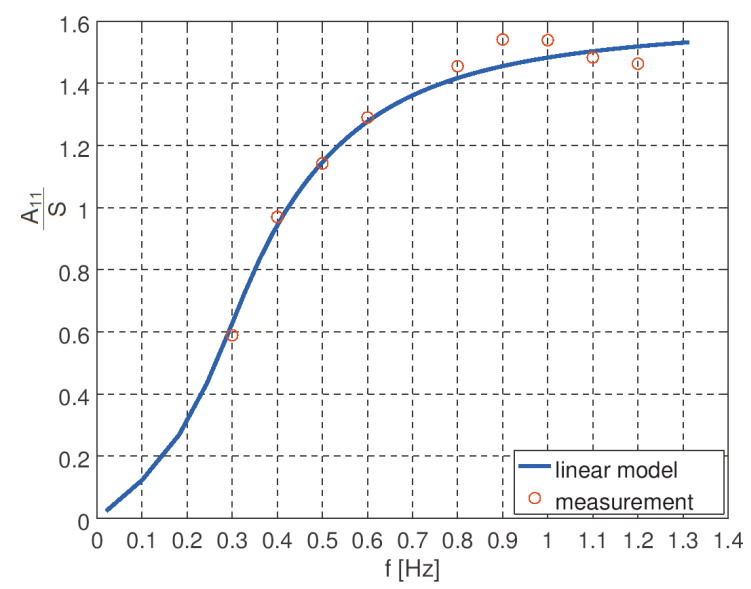

Fig. 11. The comparison between theoretical results and experimental dato for water depth $h=5.69 \mathrm{~m}$

\section{SECONDARY PHENOMENA}

In addition to the main set of measurements conducted to verify the derived model, a series of additional measurements was conducted to provide insight into the physics of secondary phenomena observed during laboratory experiments conducted in the towing tank. The profiles of regular waves were measured 
along the towing tank for the waves of the heights $2.5 \mathrm{~cm}$, $5 \mathrm{~cm}$, and $10 \mathrm{~cm}$, and frequencies $f$ from $0.3 \mathrm{~Hz}$ to $1.2 \mathrm{~Hz}$ with increment of $0.1 \mathrm{~Hz}$. The secondary phenomena were investigated and analyzed using FFT.

\section{DISINTEGRATION OF WAVE PROFILE}

The measurements were conducted by a gauge mounted on the carriage moving towards the wavemaker and then backwards. The measurements allowed to calculate the spectrum spread factor $s$. This factor has been calculated by dividing the energy of the main wave by the energy of the entire wave spectrum. The value of $s$ has been determined along the towing tank for three groups of wave frequencies $f$. The results averaged for five towing tank segments are presented in Fig. 12. It can be seen that for $f$ smaller than $1.0 \mathrm{~Hz}, s$ is constant while it is dropping along the towing tank for $f$ from 1.0 Hz. It arises from phenomenon of disintegration of wave profile and decay of main wave frequency into other frequency bands. This phenomenon increases strongly with an increase $f$ from $1.0 \mathrm{~Hz}$ to $1.2 \mathrm{~Hz}$.

\section{WAVE REFLECTIONS FROM THE BEACH}

During the measurements, the carriage was moving from the beach towards the wavemaker to capture the reflection from the rubble-mound beach. The reflected wave height and the incident wave height were extracted from the recorded signals in frequency domain by means of the Doppler shift for known velocity of the towing carriage. This allowed to identify beach reflection properties. The reflection coefficient $R$ is the ratio of the height of the reflected wave divided by the height of the incident wave. The $R$ was periodic along the test section and it was changing slightly. The value of $R$ has been averaged along the towing tank test section for investigated incident wave frequencies. The results are shown in Fig. 13. It can be seen that for investigated rubble-mound beach, $R$ is exponentially growing in the range from ca.10\% to ca. $30 \%$ in function of $f$.

\section{WAVE DAMPING}

The measurements were carried out at both ends of the deepwater towing tank during generation of regular waves of $5 \mathrm{~cm}$ height and frequencies from $0.3 \mathrm{~Hz}$ to $1.2 \mathrm{~Hz}$ with increment of $0.1 \mathrm{~Hz}$. The wave gauge was mounted on a towing carriage. The separate series of measurements were conducted on the up-wave and down-wave side of the towing tank. This technique allowed to determine wave damping along the towing tank. The relative difference between wave height on the up-wave and down-wave side of the towing tank with respect to the wave height on the up-wave side, $D$, is shown Fig. 14. It can be seen that damping of waves along the towing tank is exponentially growing with $f$ from ca. $5 \%$ damping for $f=0.4 \mathrm{~Hz}$ up to ca. $85 \%$ damping for $f=1.0 \mathrm{~Hz}$ while the waves with f equal to $1.1 \mathrm{~Hz}$ and $1.2 \mathrm{~Hz}$ get almost completely damped.

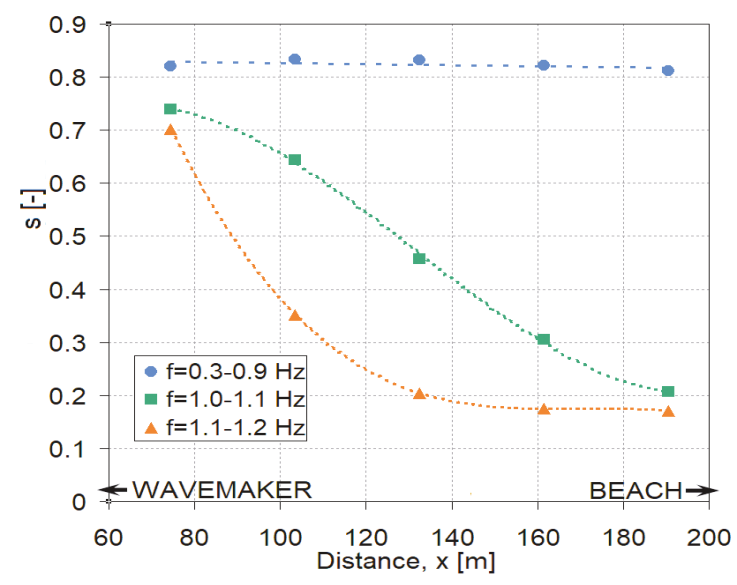

Fig. 12. The spectrum spread factor along the towing tank

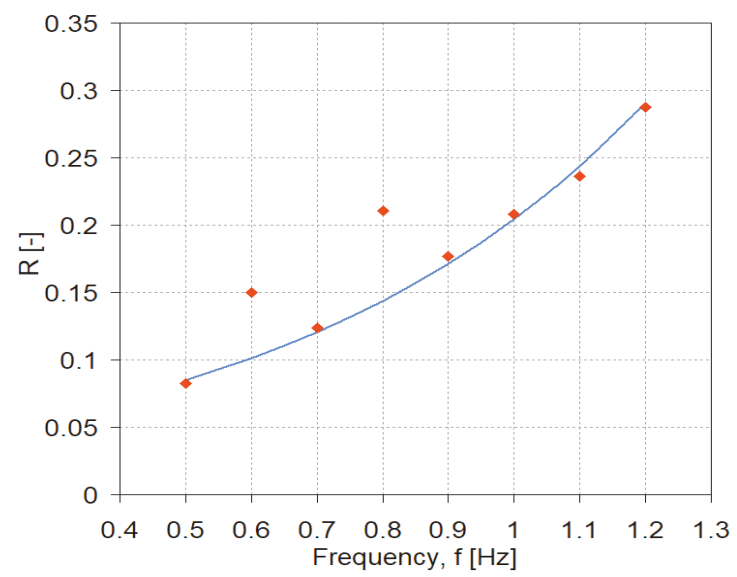

Fig. 13. Reflection coefficient versus wave frequencies for the rubble-mound beach

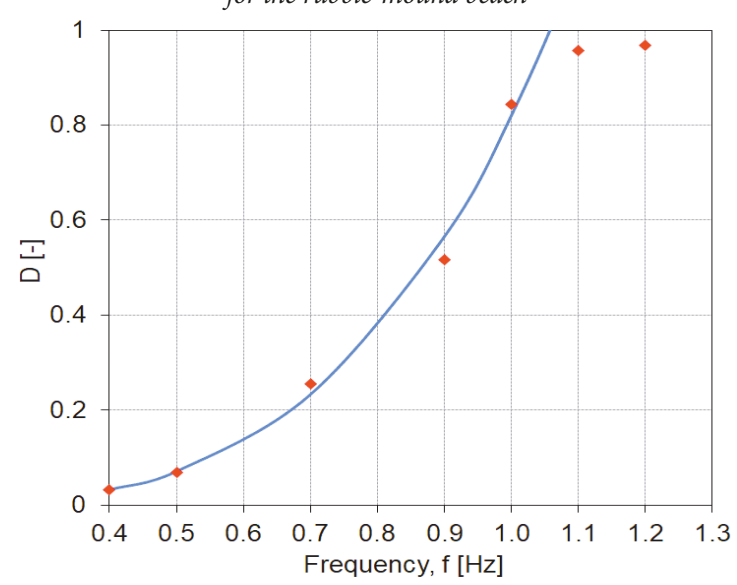

Fig. 14. Wave damping in the towing tank

\section{CONCLUSIONS}

A theoretical approach was applied to predict the generation and propagation of nonlinear water waves in a towing tank. The formulated boundary-value problem was solved by applying an analytical method. The derived model was applied to determine linear and nonlinear components of the free-surface oscillations along the towing tank.

The analysis of the results shows that for waves of very low steepness, the nonlinear wave effects and effects associated with the interaction of water waves in a generated wave train 
are of secondary importance and a linear solution may be accepted to predict waves in a towing tank. However, the discrepancies between wave profiles predicted by applying the linear and nonlinear models rapidly increases with increasing wave steepness. The analysis shows that for waves of moderate steepness the nonlinear wave component may exceed many times its linear counterpart. The analysis indicates that this phenomenon is the result of a wave generation system that generates significant components of nonlinear free waves for a wide rage of wave frequencies.

Laboratory experiments were conducted in the towing tank to verify theoretical results. The free-surface elevation recorded by a wave gauge was compared with the results provided by the derived model. Fairly good agreement between theoretical results predicted by the linear model and experimental data is observed for a wide range of wave frequencies.

In addition to the main set of measurements conducted to verify the derived model, a series of additional measurements was conducted to provide insight into the physics of secondary phenomena observed during laboratory experiments conducted in the towing tank. The secondary phenomena affect the propagation and transformation of waves along the towing tank. Knowledge on the nonlinear processes and phenomena observed in the towing tank is essential for the modeling processes.

Future work should include verification of the nonlinear model, especially the presence of significant components of nonlinear free waves for the wave generation system in the deepwater towing tank.

\section{ACKNOWLEDGEMENT}

The research was financed by the Ministry of Science and Higher Education of the Republic of Poland and by Ship Design and Research Centre CTO S.A.

\section{REFERENCES}

1. Biesel F. and Suquet F.: Laboratory wave generating apparatus. Project Report No. 39, Minneapolis 1953.

2. Campos C., Silveira F. and Mendes M.: Waves inducted by non-permanent paddle movements. Coastal Engineering Proceedings, vol. 13, 1972 pp. 707-722.

3. Drzewiecki M.: Digital Control System of the Wavemaker in the Towing Tank. Automatyka, elektryka, zakłócenia, vol. 7, no. 4, 2016, pp. 138-146.

4. Drzewiecki M.: Modelling, Simulation and Optimization of the Wavemaker in a Towing Tank. Trends in Advanced Intelligent Control, Optimization and Automation. Springer International Publishing AG, 2017, pp. 570-579.

5. Galvin C. J.: Wave-height prediction for wave generators in shallow water. Technical Memorandum No. 4, U.S. Army Corps of Engineers, 1964, Washington, pp. 1-20.
6. Grilli S. and Horrillo J.: Numerical Generation and Absorption of Fully Nonlinear Periodic Waves. Journal of Engineering Mechanics, 123(10), 1997, pp. 1060-1069.

7. Havelock T. H.: Forced surface-wave on water. Philosophical Magazine, 7, 1929, pp. 569-576.

8. Hudspeth R. T. and Sulisz W.: Stokes drift in 2-D wave flumes. Journal of Fluid Mechanics, 230, 1991, pp. 209-229.

9. Keating T. and Webber N. B.: The generation of periodic waves in a laboratory channel; a comparison between theory and experiment. Proceedings of the Institution of Civil Engineers, 63, 1977, pp. 819-832.

10. Liu S-X., Teng B. and Yu Y-X.: Wave generation in a computation domain. Applied Mathematical Modelling, 29, 2005, pp. 1-17.

11. Liu X., Lin P. and Shao S.: ISPH wave simulation by using an internal wave maker. Coastal Engineering, 95, 2015, pp. 160-170.

12. Madsen O. S.: On the generation of long waves. Journal of Geophysical Research, 76, 1971, pp. 8672-8683.

13. Moubayed W. I. and Williams A. N.: Second-order bichromatic waves produced by a generic planar wavemaker in a two-dimensional wave flume. Journal of Fluids and Structures, 8, 1994, pp. 73-92.

14. Multer R H.: Exact nonlinear model of wave generator. Journal of Hydraulic Research, ASCE, 99, 1973, pp. 31-46.

15. Schaffer H. A.: Second-order wavemaker theory for irregular waves. Ocean Engineering, 23, 1996, pp. 47-88.

16. Sulisz W. and Hudspeth R. T.: Complete second order solution for water waves generated in wave flumes. Journal of Fluids and Structures, 7(3), 1993, pp. 253-268.

17. Sulisz, W.: Diffraction of nonlinear waves by founded horizontal rectangular cylinder. Journal of Waterway, Port, Coastal, and Ocean Engineering, ASCE, 124, 5, pp. 257-263.

18. Sulisz, W.: Reflection and transmission of nonlinear water waves at a semi-submerged dock. Archives of Mechanics, 65,3 , pp. 237-260.

19. Ursell F., Dean R. G. and Yu Y. S.: Forced small amplitude waves: A comparison of theory and experiment. Journal of Fluid Mechanics, 7, 1960, pp. 33-52.

20. Wehausen J.V. and Laitone,F.V.: Surface Waves, Handbuch Der Physik. Vol. 9, Springer-Verlag, Berlin 1960. 
21. Zhang X. T., Khoo B. C. and Lou. J.: Wave propagation in a fully nonlinear numerical wave tank: A desingularized method. Ocean Engineering, 33, 2006, pp. 2310-2331

22. Zheng J., Soe M. M., Zhang C. and Hsu T-W.: Numerical wave flume with improved smoothed particle hydrodynamics. Journal of Hydrodynamics, 22(6) 2010, pp. 773-781

\section{CONTACT WITH THE AUTHORS}

Marcin Drzewiecki

e-mail:drzewiecki.marcin@op.pl

Gdańsk University of Technology

Narutowicza 11/12

80-233 Gdańsk

Ship Deign and Research Centre CTO S.A.

Szczecińska 65

80-392 Gdańsk

PolAND

\section{Wojciech Sulisz}

e-mail:sulisz@ibwpan.gda.pl

Institute of Hydroengineering

of Polish Academy of Sciences IBW PAN

Kościerska 7

80-328 Gdansk

Poland 